2018

\title{
Dielectric Properties of Isolated Adrenal Chromaffin Cells Determined by Microfluidic Impedance Spectroscopy
}

\author{
A. C. Sabuncu
}

M. Stacey

Old Dominion University, mstacey@odu.edu

G. L. Graviso

N. Semenova

Old Dominion University

P. T. Vernier

Old Dominion University, pvernier@odu.edu

See next page for additional authors

Follow this and additional works at: https://digitalcommons.odu.edu/bioelectrics_pubs

Part of the Biochemistry Commons, Biomedical Engineering and Bioengineering Commons, $\underline{\text { Biophysics Commons, Biotechnology Commons, and the Molecular Biology Commons }}$

\section{Repository Citation}

Sabuncu, A. C.; Stacey, M.; Graviso, G. L.; Semenova, N.; Vernier, P. T.; Leblanc, N.; Chatterjee, I.; and Zaklit, J., "Dielectric Properties of Isolated Adrenal Chromaffin Cells Determined by Microfluidic Impedance Spectroscopy" (2018). Bioelectrics Publications. 214.

https://digitalcommons.odu.edu/bioelectrics_pubs/214

\section{Original Publication Citation}

Sabuncu, A. C., Stacey, M., Craviso, G. L., Semenova, N., Vernier, P. T., Leblanc, N., ... Zaklit, J. (2018). Dielectric properties of isolated adrenal chromaffin cells determined by microfluidic impedance spectroscopy. Bioelectrochemistry, 119, 84-91. doi:10.1016/ j.bioelechem.2017.09.001 
Authors

A. C. Sabuncu, M. Stacey, G. L. Graviso, N. Semenova, P. T. Vernier, N. Leblanc, I. Chatterjee, and J. Zaklit 


\title{
Dielectric properties of isolated adrenal chromaffin cells determined by microfluidic impedance spectroscopy
}

\author{
Authors \\ A C Sabuncu ${ }^{1, *}$, M Stacey ${ }^{2}, \mathrm{G}$ L Craviso ${ }^{3}$, N Semenova ${ }^{2}$, P T Vernier ${ }^{2}$, N Leblanc ${ }^{3}$, I Chatterjee ${ }^{4}$ and J \\ Zaklit $^{4}$ \\ ${ }^{1}$ Department of Mechanical Engineering, Southern Methodist University, Dallas, TX 75205, USA \\ ${ }^{2}$ Frank Reidy Research Center for Bioelectrics, Old Dominion University, Norfolk, VA 23508, USA \\ ${ }^{3}$ Department of Pharmacology, University of Nevada, Reno School of Medicine, Reno, NV 89557, USA \\ ${ }^{4}$ Department of Electrical and Biomedical Engineering, College of Engineering, University of Nevada, \\ Reno, Reno, NV 89557, USA \\ ${ }^{*}$ Contact author. E-mail: asabuncu@smu.edu
}

Corresponding author: Ahmet Can Sabuncu

Email: asabuncu@smu.edu

Phone: 2147683200

Fax: 214-768-1473

\begin{abstract}
Knowledge of the dielectric properties of biological cells plays an important role in numerical models aimed at understanding how high intensity ultrashort nanosecond electric pulses affect the plasma membrane and the membranes of intracellular organelles. To this end, using electrical impedance spectroscopy, the dielectric properties of isolated, neuroendocrine adrenal chromaffin cells were obtained. Measured impedance data of the cell suspension, acquired between $1 \mathrm{kHz}$ and $20 \mathrm{MHz}$, were fit into a combination of constant phase element and Cole-Cole models from which the effect of electrode polarization was extracted. The dielectric spectrum of each cell suspension was fit into a Maxwell-Wagner mixture model and the Clausius-Mossotti factor was obtained. Lastly, to extract the cellular dielectric parameters, the cell dielectric data were fit into a granular cell model representative of a chromaffin cell, which was based on the inclusion of secretory granules in the cytoplasm. Chromaffin cell parameters determined from this study were the cell and secretory granule membrane specific capacitance (1.22 and $7.10 \mu \mathrm{F} / \mathrm{cm}^{2}$, respectively), the cytoplasmic conductivity, which excludes and includes the effect of
\end{abstract}


intracellular membranous structures (1.14 and $0.49 \mathrm{~S} / \mathrm{m}$, respectively), and the secretory granule milieu conductivity $(0.35 \mathrm{~S} / \mathrm{m})$. These measurements will be crucial for incorporating into numerical models aimed at understanding the differential poration effect of nanosecond electric pulses on chromaffin cell membranes.

\section{Keywords}

Dielectric spectroscopy; Impedance measurement; Chromaffin cells; Dielectric cell modeling;

Permittivity; Conductivity; Membrane capacitance 


\section{Introduction}

Electric pulse exposure of biological cells causes electroporation of the plasma membrane, allowing molecules typically unable to penetrate cell membranes, such as DNA or peptides, to gain access to the cell interior (Chang and Reese, 1990). For electric pulses that are nanoseconds in duration, cell responses include both permeabilization of the plasma membrane (Vernier et al., 2006, Pakhomov et al., 2007a, Pakhomov et al., 2007b, Pakhomov et al., 2009) and permeabilization of the membranes of internal organelles such as the endoplasmic reticulum (Scarlett et al., 2009, Semenov et al., 2013a, Semenov et al., 2013b.), the latter the result of the electric field penetrating into the cell interior (Schoenbach et al., 2001). Studies exploring this latter effect have been numerous, driven by the potential for nanosecond electric pulses to manipulate cellular function in new ways (Schoenbach et al., 2001). Included in this research effort is the use of numerical models to understand the electroporation effects of these pulses on cellular membranes (Gowrishankar et al., 2006; Smith and Weaver, 2008).

Numerical models take into account the specific electric field parameters of the pulse applied to a cell, such as pulse shape, pulse duration and electric field amplitude, as well as the dielectric properties of the cell membrane, cytoplasm and intracellular membranous structures. However, these dielectric properties are typically unknown for the particular cell type that is under experimental investigation. Thus, the values for the dielectric properties used in the models are usually those reported for other cell types (Gowrishankar et al., 2006; Smith and Weaver, 2008). One consequence is that numerical modeling predictions of permeabilization of membranes of relevant internal organelles, which typically include the nucleus, mitochondria and endoplasmic reticulum, may not agree with actual experimental findings. Our investigations of the effect of 5-ns, 5-6 MV/m pulses on isolated adrenal chromaffin cells, a well-established model of neurosecretion, provide such an example. We found that the experimentally determined electric field amplitude that is required to cause intracellular membrane permeabilization is at least twice as great as that for causing plasma membrane permeabilization. In contrast, our numerical modeling results predicted a similar electric field threshold for electroporation of chromaffin cell membranes regardless of their location, that is, intracellular versus the plasma membrane (Zaklit et. al., 2015; Zaklit et. al., 2017). 
Clearly, knowledge of the actual dielectric properties of chromaffin cells should enable better agreement between cell modeling simulations and the experimental findings. This point takes on added significance since adrenal chromaffin cells contain, in addition to a nucleus, mitochondria and endoplasmic reticulum, a multitude of membrane-delineated secretory granules (7,000 to 41,400 per cell, with an average of 23,500 granules per cell; (Plattner et al., 1997; Huh et al., 2005a; Huh et al., 2005b) in their cytoplasm that could play a role in how the nanosecond-duration electric field affects internal organelle membranes.

The goal of this work was to measure the dielectric properties of adrenal chromaffin cells using dielectric spectroscopy, a well-accepted non-invasive method for measuring the AC permittivity and conductivity of biological cells and their intracellular membranous structures. In this method, a small voltage is applied to a cell suspension contained between two electrodes, and the dielectric spectrum over a range of frequencies obtained by measuring the resultant current. Here we describe how the dielectric properties of chromaffin cells and their intracellular secretory granules were derived using dielectric spectroscopy in combination with a constant phase element, Cole-Cole, Maxwell-Wagner mixture models and a granular cell model.

\section{Methods}

\subsection{Isolation and preparation of adrenal chromaffin cells}

Fresh bovine adrenal glands were obtained from a local abattoir (Wolf Pack Meats, Reno, NV) and chromaffin cells were isolated from the medulla by collagenase digestion as described previously (Waymire et al., 1983; Hassan et al., 2002). Cells were maintained in suspension culture at $36.5^{\circ} \mathrm{C}$ under a humidified atmosphere of $5 \% \mathrm{CO}_{2}$. The large aggregates of cells that form in suspension culture were enzymatically dissociated into single cells with the protease dispase (Craviso, 2004) prior to dielectric spectroscopy measurement. For the impedance measurements, the cells were resuspended at variable volume fractions (5.7 to $11.1 \%$, table 1 ) in a physiological salt solution (PSS) of the following composition: $145 \mathrm{mM} \mathrm{NaCl}$, $5 \mathrm{mM} \mathrm{KCl}, 1.2 \mathrm{mM} \mathrm{NaH}_{2} \mathrm{PO}_{4}, 2 \mathrm{mM} \mathrm{CaCl} 2,1.3 \mathrm{mM} \mathrm{MgCl}_{2}, 10 \mathrm{mM}$ glucose and $15 \mathrm{mM}$ HEPES, pH 7.4. 
a

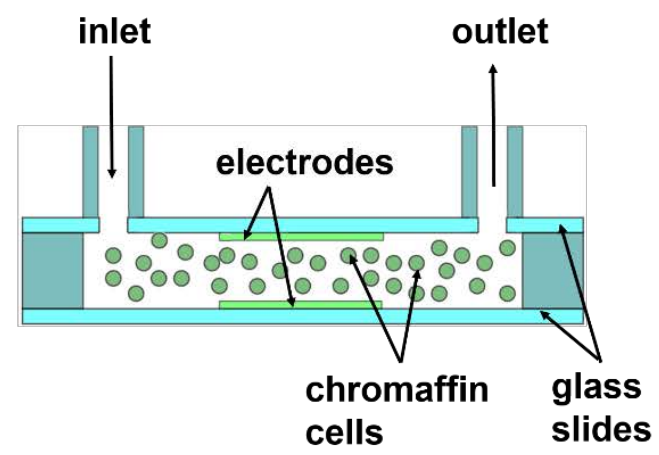

b

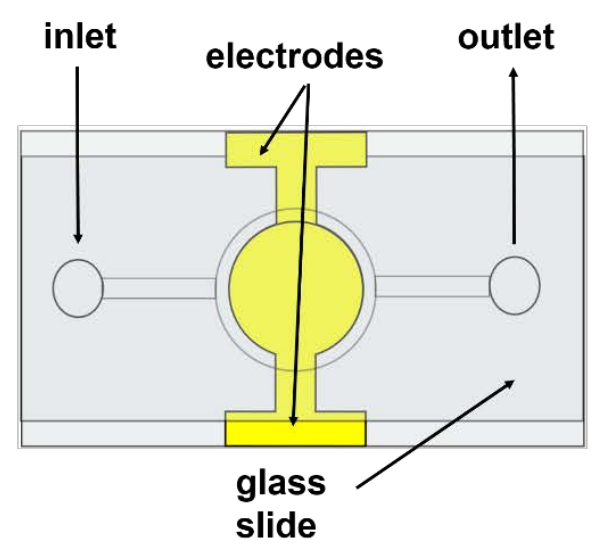

C

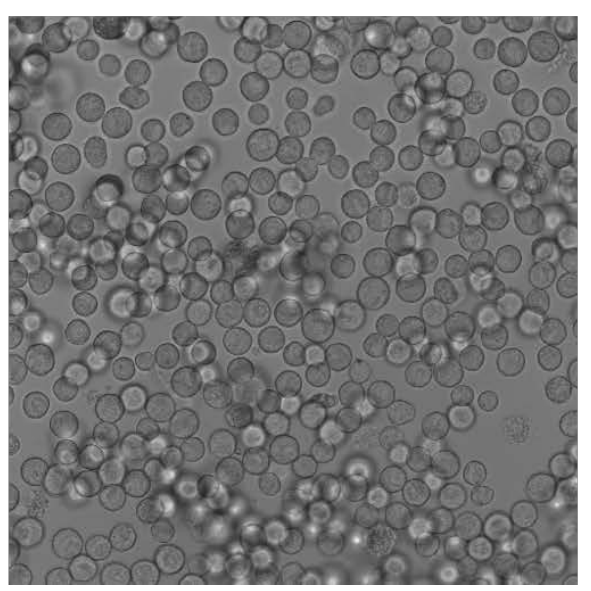

Figure 1 Details of the microfluidic chamber used to make the dielectric spectroscopy measurements on chromaffin cells. Shown in (a) and (b) are schematics of the side-view and top-view, respectively, of the microfluidic chamber. Shown in (c) is a photomicrograph (40X) of the chromaffin cell suspensions used in the measurements. 


\subsection{Microfluidic chamber fabrication}

Figures $1 \mathrm{a}$ and $1 \mathrm{~b}$ show a schematic of the microfluidic chamber used to measure the impedance spectrum of chromaffin cells. The chamber consisted of two parallel glass plates, each containing a circular gold electrode (radius $=2 \mathrm{~mm}$ ). The electrodes were aligned on top of each other and the glass plates joined using $250 \mu \mathrm{m}$ thick double-sided tape, creating a chamber of height $250 \mu \mathrm{m}$. Details for the fabrication of the chamber, which are given in a previous paper (Koklu et al., 2016), are briefly described here. Glass microscope slides were cut into $2.5 \times 2.5 \mathrm{~cm}^{2}$ pieces using a diamond cutter and inlet and outlet ports created by boring holes into the glass using a diamond drill bit. Glass pieces were cleaned sequentially in an ultrasonic bath with deionized (DI) water, $1 \mathrm{M} \mathrm{KOH}$, acetone, followed by a rinse with DI water. The glass slides, purged with a stream of nitrogen gas, were dried in a convection oven at $120^{\circ} \mathrm{C}$ for $10 \mathrm{~min}$. Masking tape was then applied to the glass surface as a negative mask to generate the desired circular electrode pattern. Chromium was sputter deposited (EMS300TD, Emitech; 120 mA-60 s) onto the glass to serve as a seeding layer for the subsequent deposition of gold, which was sputter deposited (120 mA-150 s) to a thickness of $75 \mathrm{~nm}$. Because impedance measurements for chromaffin cells would be made in a high conductivity ( $\sim 1 \mathrm{~S} / \mathrm{m})$ medium, the surface topology of the electrodes was modified by electrochemical deposition of gold using a three-electrode potentiostat/galvanostat system (EZstatPro, Nuvant). This step, which increases surface area by generating fractal structures, was necessary to minimize the destructive effects of electrode polarization, i.e., charge accumulation at the electrode/electrolyte interface that can cause orders of magnitude larger resistance and capacitance than the bulk liquid as ions close to the energized electrodes form an electrical double layer. Thus, by increasing the electrode surface area in contact with the cell suspension, the electrode polarization shifted to lower frequencies, leading to an observable dielectric dispersion signal from the cell suspension (Schwan, 1968; Pajkossy, 1994).

\subsection{Impedance spectroscopy}

The electrodes of the microfluidic chamber connected to an impedance analyzer (4294A, Agilent) via a BNC port. The impedance analyzer interfaced with a computer that acquires the raw measured impedance 
data, recorded in the frequency range of $1 \mathrm{kHz}$ to $20 \mathrm{MHz}$. Details about the impedance measurement system, the calibration procedure including elimination of the effects of cables and the test fixture are available in (Sabuncu et al., 2012). Data were collected in triplicate for each independent experiment and expressed as mean \pm standard deviation (SD).

To make the dielectric spectroscopy measurements, chromaffin cells were suspended in the PSS described previously (conductivity $=1.35 \mathrm{~S} / \mathrm{m}$ ) and a $10 \mu \mathrm{l}$ aliquot of the cell suspension was fed into the empty chamber at the inlet port using a pipette. The measurement volume was $3.1 \mu \mathrm{l}$ and the remaining portion of the aliquot filled the connecting inlet and outlet channels. For each replication, a new cell suspension was aliquoted into the chamber. Figure 1c shows a bright field image of the cells prior to being loaded into the chamber. Measurement of the impedance spectrum of the cell suspension was obtained during the static state, that is, after the initial flow generated by the introduction of the cell suspension into the fluidic channel had stopped. Application of a small test voltage $(0.5 \mathrm{~V})$ to the electrodes of the chamber allowed measurement of cell suspension impedance as magnitude and phase angle.

Details of the derivation of the cell complex permittivity from the bulk impedance are in a previous publication (Sabuncu et al., 2012) and briefly described here. First, calculation of the dielectric spectrum of the cells using the unit and stray capacitance of the microfluidic chamber, the impedance measurement of the chamber when empty or filled with DI water, and the measured impedance data, was made. Second, the dielectric spectrum of the cells was fit into a combination of a constant phase element and Cole-Cole models to extract the effects of electrode polarization and lead impedance (Koklu et al., 2016). The lead resistance and inductance of the impedance device were determined using PSS impedance measurements between 10 and $20 \mathrm{MHz}$. The lead resistance and inductance were changed in a fitting routine until the conductivity found using the device matched the conductivity measured using a conductivity meter (Orion Star $^{\mathrm{TM}}$ A322, Fisher Scientific). Third, the dielectric spectrum of the cell suspension was fit into a MaxwellWagner mixture model to extract the Clausius-Mossotti factor, which is a function of the cell complex permittivity. Fourth, the cell complex permittivity was calculated using the extracted Clausius-Mossotti factor, a granular cell model (described below), and external medium (i.e., PSS) properties. 


\subsection{Granular cell model}

To represent chromaffin cells that contain a large volume fraction of secretory granules in their cytoplasm, we used a vesicular cell model that was originally used by Raicu et al. (Raicu et al., 1998) for modeling hepatocytes that contain a large volume fraction (30\%) of mitochondria in the cytosol (Irimajiri et al., 1991). A schematic for the vesicular model (referred to in this paper as the granular cell model) is shown in figure 2. Two circular concentric shells representing the cell membrane and nuclear envelope are separated by a suspension of granules. According to the model, the cell complex permittivity is given by:

$\varepsilon_{\text {cell }}^{*}=\varepsilon_{m e m}^{*} \frac{2\left(1-v_{m}\right) \varepsilon_{m e m}^{*}+\left(1+2 v_{m}\right) \varepsilon_{i n t}^{*}}{\left(2+v_{m}\right) \varepsilon_{m e m}^{*}+\left(1-v_{m}\right) \varepsilon_{i n t}^{*}}$

where subscripts mem and int stand for cell membrane and cell interior, respectively. In the above equation, $v_{m}=[1-d / R]^{3}$ where $d$ is the cell membrane thickness and $R$ is the cell radius. The complex permittivity of the cell interior or cytosol is given by:

$\varepsilon_{i n t}^{*}=\varepsilon_{I S}^{*} \frac{2\left(1-v_{n}\right) \varepsilon_{I S}^{*}+\left(1+2 v_{n}\right) \varepsilon_{N}^{*}}{\left(2+v_{n}\right) \varepsilon_{I S}^{*}+\left(1-v_{n}\right) \varepsilon_{N}^{*}}$

where subscripts $N$ and $I S$ stand for the nucleus and the material between the cell membrane and the nucleus, respectively. In the above equation, $v_{n}=\left[R_{N} /(R-d)\right]^{3}$ where $R_{N}$ is the radius of the nucleus. The complex permittivity of the nucleus is given by:

$\varepsilon_{N}^{*}=\varepsilon_{n m}^{*} \frac{2\left(1-v_{n m}\right) \varepsilon_{n m}^{*}+\left(1+2 v_{n m}\right) \varepsilon_{n p}^{*}}{\left(2+v_{n m}\right) \varepsilon_{n m}^{*}+\left(1-v_{n m}\right) \varepsilon_{n p}^{*}}$

where subscripts $\mathrm{nm}$ and $\mathrm{np}$ stand for nuclear envelope and nucleoplasm, respectively. In the above equation, $v_{n m}=\left[1-d_{N} / R_{N}\right]^{3}$ where $d_{N}$ is the nuclear envelope thickness. The complex permittivity of the material between the nucleus and the cell membrane is given by the following equation that also accounts for the granules in the cytoplasm:

$\varepsilon_{I S}^{*}=\varepsilon_{c y t}^{*} \frac{2\left(1-v_{g r n}\right) \varepsilon_{c y t}^{*}+\left(1+2 v_{g r n}\right) \varepsilon_{g r n}^{*}}{\left(2+v_{g r n}\right) \varepsilon_{c y t}^{*}+\left(1-v_{g r n}\right) \varepsilon_{g r n}^{*}}$

where subscripts $c y t$ and $g r n$ stand for cytoplasm and granules, respectively. In the above equation, $v_{g r n}$ is the volume fraction of granules in the region between the nucleus and the cell membrane. The complex permittivity of a granule is given by: 
$\varepsilon_{g r n}^{*}=\varepsilon_{g m}^{*} \frac{2\left(1-v_{g m}\right) \varepsilon_{g m}^{*}+\left(1+2 v_{g m}\right) \varepsilon_{g i}^{*}}{\left(2+v_{g m}\right) \varepsilon_{g m}^{*}+\left(1-v_{g m}\right) \varepsilon_{g i}^{*}}$

where subscripts $g m$ and $g i$ stand for granule membrane and granule milieu, respectively. In the above equation, $v_{g m}=\left[1-d_{g r n} / R_{g r n}\right]^{3}$ where $d_{g r n}$ and $R_{g r n}$ are the granule membrane thickness and granule radius, respectively. The above equations essentially describe a simplified cell model where the complex permittivities of internal organelles and the cytosol are replaced by an effective complex permittivity term, which has frequency-independent permittivity and conductivity terms. Once the permittivity spectrum of the cell complex was found, the equivalent cell suspension permittivity was calculated through the Maxwell-Wagner mixture model (Sabuncu et al., 2012) that is given by:

$\varepsilon_{s u s}^{*}=\varepsilon_{m e d}^{*} \frac{1+2 p f_{c m}}{1-p f_{c m}}$

where subscripts sus and med stand for cell suspension and extracellular medium, respectively. In the above equation $f_{c m}$ is the Clausius-Mossotti factor, and $p$ stands for volume fraction of the cells. Scripts written in MATLAB ${ }^{\circledR}$ (2016a, Mathworks) were used to extract the dielectric properties of chromaffin cells and their intracellular granules.

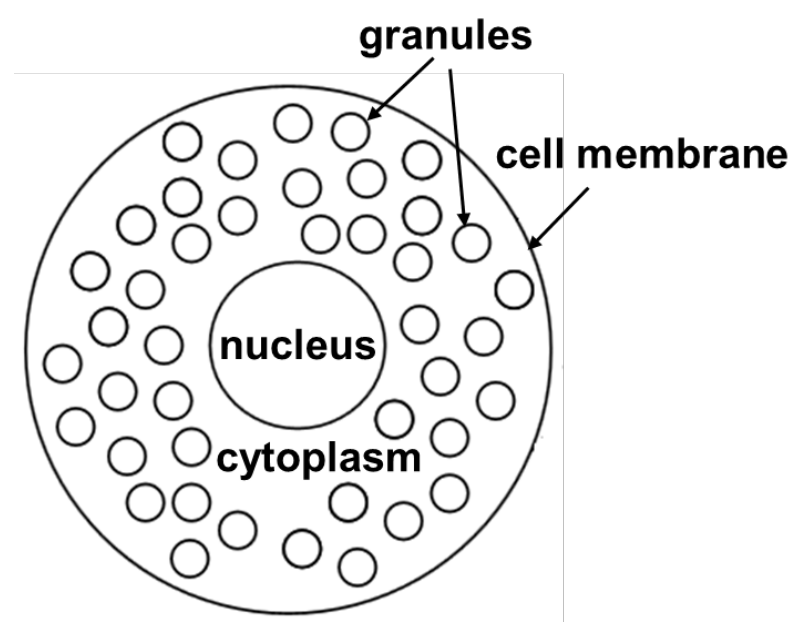

Figure 2 Schematic of the granular cell model. The model includes a cell membrane, cytoplasm, nucleus, and multiple granules in the cytoplasm. 


\subsection{Determination of the fit parameters}

The dielectric spectra of the cell suspension were calculated using equations (1) to (6), which are functions of the cell dielectric properties, specifically the complex permittivities of the plasma membrane, granule membrane, granule milieu, cytoplasm, nuclear envelope, and nucleoplasm. The experimental data, on the other hand, include the impedances of the cell suspension, electrode/electrolyte interface, and the stray effects. Once the unit and stray capacitances of the measurement chamber were determined, the experimental impedance data were simulated using the cell model.

A sensitivity analysis was performed to determine which cellular dielectric parameters could accurately be extracted from the experimental impedance data. In their current form, equations (1) to (6) have 22 variables, which include the volume fraction of cells in suspension, volume fraction of granules in the cytosol, geometrical and dielectric properties of intracellular components and extracellular medium. The fitting of equations (1) to (6) to experimental impedance data in the interfacial ( $\beta$ ) dispersion range, which occurs due to cell membrane charging, represents an underdetermined system where the number of unknowns (22) exceeds the total number of variables that are solvable. Given the experimental impedance data, the interfacial polarization could be characterized by six parameters including the extracellular conductivity. Therefore, we decreased the number of unknowns in the system by fixing some parameters either to independently measured or to assumed quantities whose variations do not affect the spectra (Polevaya et al., 1999). By performing the sensitivity analysis, we determined the six variables that can be accurately extracted from dielectric spectroscopy measurements. The cell dielectric properties that minimized the residual between the simulated and experimental impedance data were used to characterize chromaffin cells. A fitting algorithm was utilized to perform the optimization using the nested Isqonlin function in MATLAB to minimize the sum of the squares of the residuals, defined as:

Residual $=\left[\sum_{i}\left(1-\log \varepsilon_{t h, i} / \log \varepsilon_{m e a, i}\right)^{2}+\sum_{i}\left(1-\sigma_{t h, i} / \sigma_{m e a, i}\right)^{2}\right]^{0.5}$

where subscripts th and mea stand for theory and measurement, respectively, and subscript $i$ represents different frequency points. In the fitting routine, each parameter in equations (1) to (6), except the 
independently measured parameters such as the volume fraction of cells $(p)$ and cell radius, was varied in an expected range that was determined based on a range of reference values reported in the literature (Polevaya et al., 1999; Sabuncu et al., 2012; Stacey et al., 2014), and the resulting cell suspension dielectric spectra were analyzed. All parameters, including the geometric properties $\left(v_{m}, v_{n m}, v_{n}, v_{g m}\right)$ in equations (1) to (6), that were fixed to constant values are tabulated in table 1.

Table 1. Geometric and dielectric properties that were fixed to constant values while modeling the experimental data.

\begin{tabular}{lc}
\hline Cell Radius, $R(\mu \mathrm{m})$ & $8^{\mathrm{a}}$ \\
Cell Membrane Thickness, $d(\mathrm{~nm})$ & 5 \\
Cell Membrane Conductivity, $\sigma_{m e m}(\mathrm{~S} / \mathrm{m})$ & $8.3 \times 10^{-10}$ \\
Extracellular Water Relative Permittivity, $\sigma_{m e d}(\mathrm{~S} / \mathrm{m})$ & 80 \\
Cytoplasm Relative Permittivity, $\varepsilon_{c y t}$ & 80 \\
Volume Fraction of Cells, $p(\%)$ & $5.7,9.4,11.1^{\mathrm{b}}$ \\
Nucleus Radius, $R_{N}(\mu \mathrm{m})$ & $2.5^{\mathrm{c}}$ \\
Nuclear Envelope Thickness, $d_{N}(\mathrm{~nm})$ & 10 \\
Nuclear Envelope Relative Permittivity, $\varepsilon_{n m}$ & 10 \\
Nuclear Envelope Conductivity, $\sigma_{n m}(\mathrm{~S} / \mathrm{m})$ & $10^{-4}$ \\
Nucleoplasm Relative Permittivity, $\varepsilon_{n p}$ & 80 \\
Nucleoplasm Conductivity, $\sigma_{n p}(\mathrm{~S} / \mathrm{m})$ & 1 \\
Granule Membrane Thickness, $d_{g r n}(\mathrm{~nm})$ & 5 \\
Granule Radius, $R_{g r n}(\mu \mathrm{m})$ & 0.14 \\
Intra-granular Milieu Relative Permittivity, $\varepsilon_{g i}$ & 80 \\
Granule Membrane Conductivity, $\sigma_{g m}(\mathrm{~S} / \mathrm{m})$ & $8.3 \times 10^{-10}$ \\
\hline
\end{tabular}

${ }^{a}$ Obtained from the cell diameter measured using light microscopy.

${ }^{\mathrm{b}}$ Measured by pelleting the cell suspension in a hematocrit tube, and measuring the volume of packed cells in the tube. The values are for three independent experiments.

${ }^{\mathrm{c}}$ Obtained from (Plattner et al., 1997)

\section{Results and Discussion}

In this work, we measured the dielectric properties of chromaffin cells, which include the cell membrane capacitance, cytosol conductivity, granule membrane capacitance and granule milieu conductivity, using microfluidic dielectric spectroscopy (Sabuncu et al., 2012). To the best of our knowledge, there are no previous dielectric measurements conducted in chromaffin cells. Details on the electrode polarization parameters, impedance spectra of salt solutions, fitting algorithm, uniqueness of the fitting and Clausius-Mossotti factors are described in previous publications (Koklu et al., 2016; Sabuncu et al., 2012). 
a

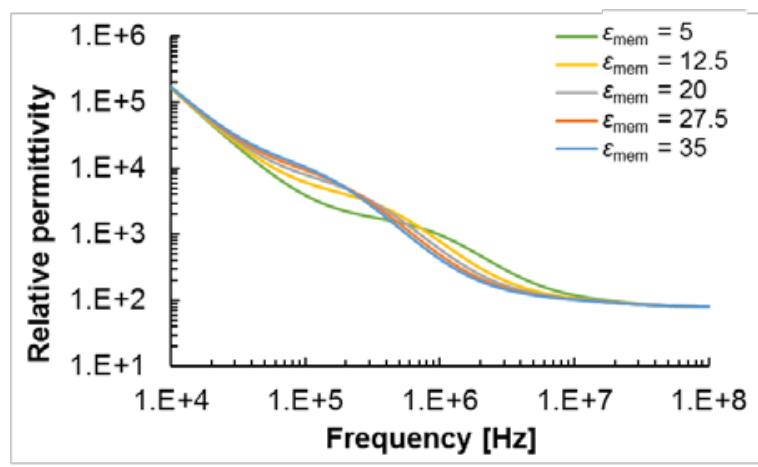

C

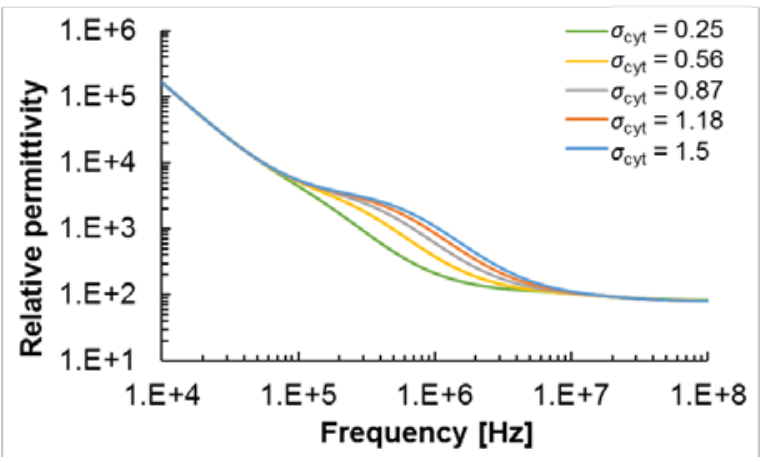

b

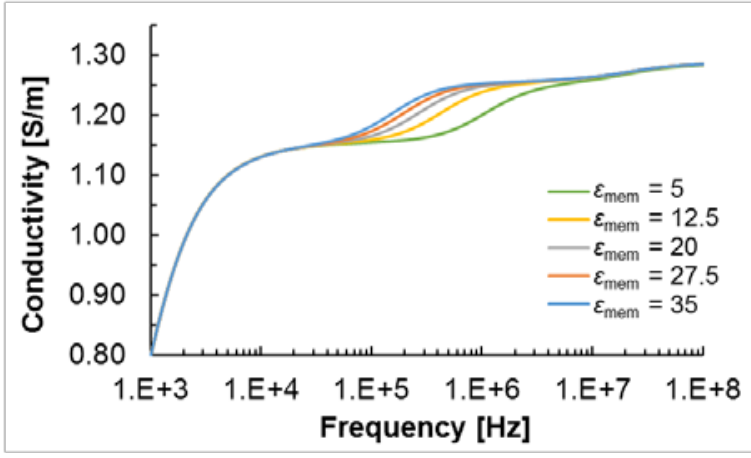

d

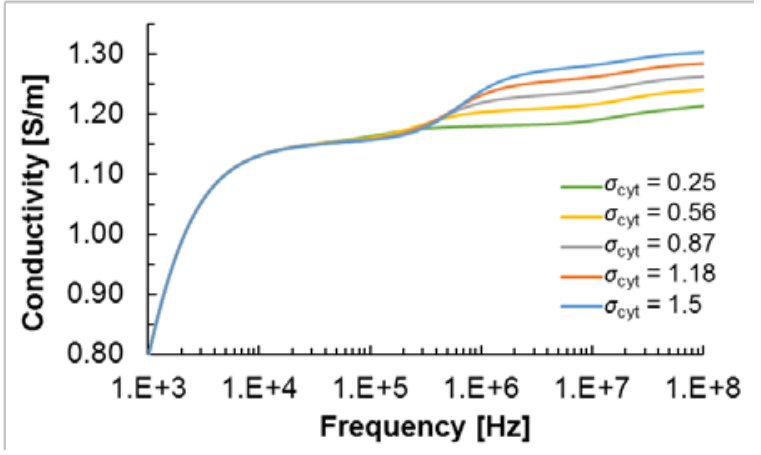

Figure 3 Changes in relative permittivity and conductivity spectra of the simulated chromaffin cell suspension with changing cell membrane and cytoplasm parameters. The cell suspension spectra were simulated using equations (1) to (6). The suspension parameters that were incrementally changed in the simulations are the cell membrane permittivity (a) and (b), and cytoplasmic conductivity (c) and (d).

\subsection{Effect of cellular dielectric properties on the dielectric spectra of the chromaffin cell suspension}

At low extracellular conductivity values $(\sim 0.01 \mathrm{~S} / \mathrm{m})$ dielectric spectroscopy has been shown to be sensitive to changes in cell membrane conductivity (Sabuncu et al., 2012; Stacey et al., 2014; Polevaya et al., 1999). However, when cells are suspended in a high conductivity medium ( 1 S/m), such as in this study, the dielectric spectra are not sensitive to changes in the conductivity of the cell membrane that is a poor conductor (conductivity less than $10^{-5} \mathrm{~S} / \mathrm{m}$ ). In fact, results obtained from the sensitivity analysis indicated that the cell membrane conductivity was a parameter that could not be extracted accurately from dielectric spectroscopy measurements performed in high conductivity media (results not shown). This result has also been demonstrated in a previous study (Beving et al., 1994). Therefore, in fitting the granular cell model to the experimental data, membrane conductivity was set to a fixed quantity. 
a

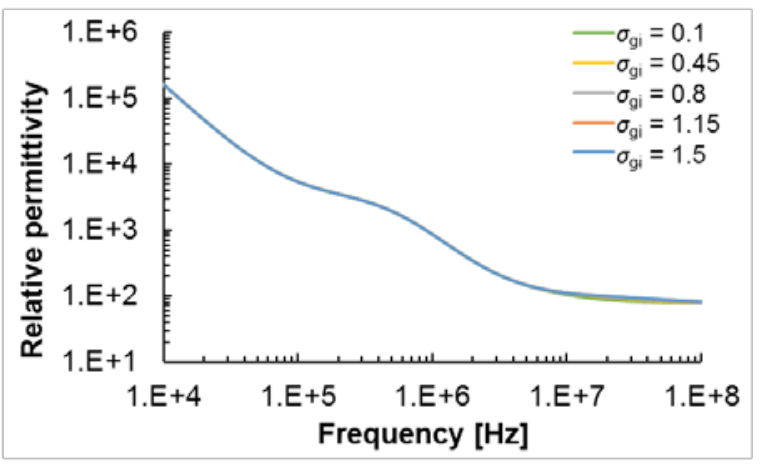

C

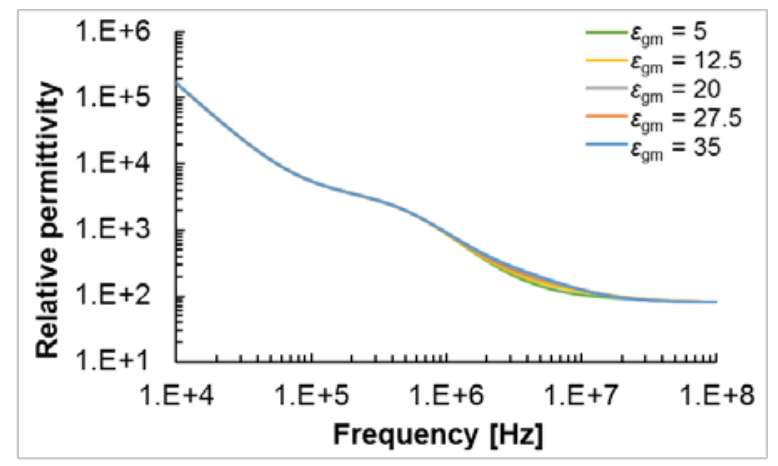

e

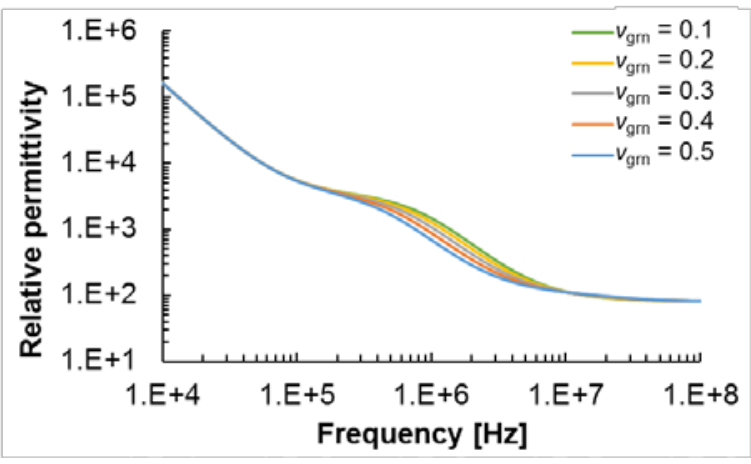

b

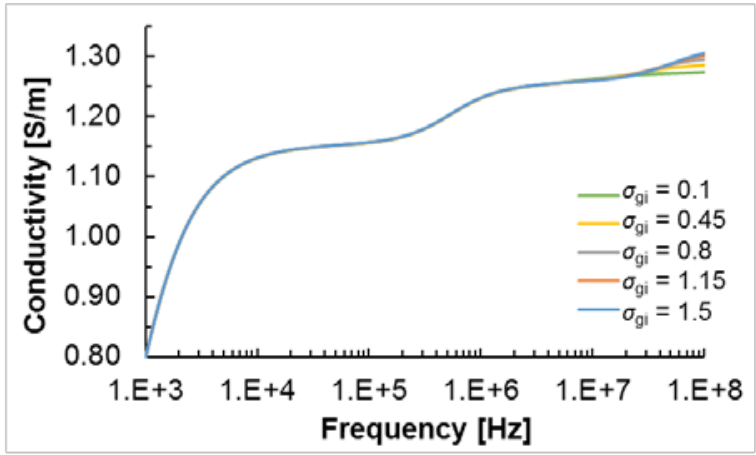

d

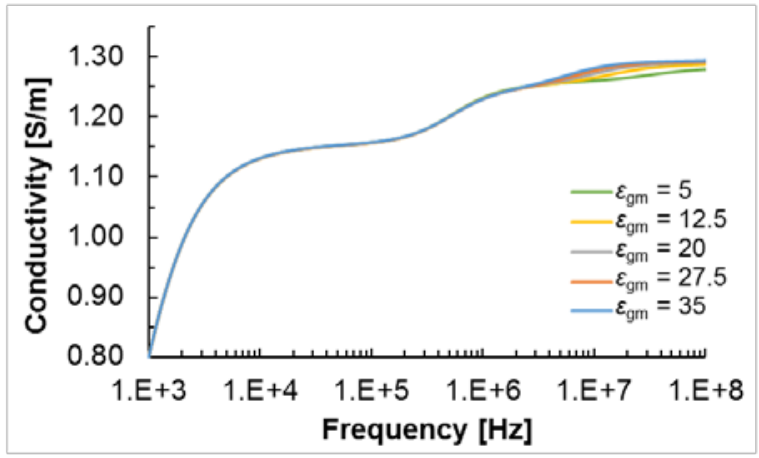

f

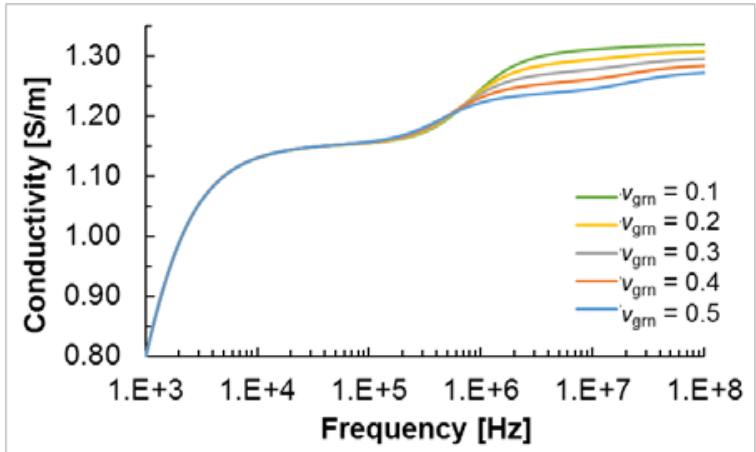

Figure 4 Changes in relative permittivity and conductivity spectra of the simulated chromaffin cell suspension with changing granule parameters. The cell suspension spectra were simulated using equations (1) to (6). The suspension parameters that were incrementally changed in the simulations are conductivity of the granule milieu (a) and (b), granule membrane permittivity (c) and (d), and granule volume fraction in the cell interior (e) and (f). The small changes in (a) and (b) are shown in the supporting information figure S1. 
Figures 3 and 4 show the effect of each parameter that had the highest influence on the dielectric spectra of the cell suspension. These parameters are the cell membrane permittivity $\left(\varepsilon_{m e m}\right)$, cytoplasmic conductivity $\left(\sigma_{c y t}\right)$, granule membrane permittivity $\left(\varepsilon_{g m}\right)$, granule milieu conductivity $\left(\sigma_{g i}\right)$ and volume fraction of granules $\left(v_{g r n}\right)$.

As shown in figures 3a and 3b, the cell membrane permittivity substantially affects the cell suspension spectra at frequencies below $10 \mathrm{MHz}$, specifically by changing the low frequency permittivity and the dielectric dispersion time constant of the cell suspension. In addition, figures 3c and 3d indicate that the cytosol conductivity also affects the time constant of the dielectric dispersion of cell suspensions similar to how the cell membrane permittivity affects the time constant of the dispersion.

The effects of changing the granule parameters on the cell dielectric spectra are shown in figure 4. These results show that while the granule milieu conductivity (figures $4 \mathrm{a}$ and $4 \mathrm{~b}$ ) and the granule membrane permittivity (figures 4c and 4d) affect the cell suspension spectra at frequencies above $10 \mathrm{MHz}$, varying the granule volume fraction (figures $4 \mathrm{e}$ and $4 \mathrm{f}$ ) affects the time constant of the dielectric dispersion of cell suspensions. In addition, varying the granule volume fraction causes an effect that is similar to that caused by varying the cytoplasmic conductivity, which is a change in the time constant of the dielectric dispersion. A zoomed-in view of the small changes in figures 4a and 4b is shown in the Supplementary material, figure S1.

Taken together, the results shown in figures 3 and 4 indicate that the cell suspension spectra are the least sensitive to changes in granule milieu conductivity and granule membrane permittivity.

\subsection{Determination of chromaffin cell parameters}

\subsubsection{Cell membrane capacitance}

The dielectric properties of chromaffin cells, obtained using the fitting algorithm, are tabulated in table 2. In this table, the membrane capacitance was calculated using the following formula: $C_{m e m}=\varepsilon_{m e m} \varepsilon_{o} / d$, where $\varepsilon_{\text {mem }}=6.8$ is the measured average cell membrane relative permittivity, $d$ is the membrane 
thickness and $\varepsilon_{o}$ is the permittivity of free space. The measured membrane capacitance value of chromaffin cells $\left(1.22 \mu \mathrm{F} / \mathrm{cm}^{2}\right)$ is close to that found for blood cells $\left(0.91\right.$ to $\left.1.62 \mu \mathrm{F} / \mathrm{cm}^{2}\right)$, breast cancer cell lines $(1.44$ to $\left.2.75 \mu \mathrm{F} / \mathrm{cm}^{2}\right)$, and a melanoma cell line $\left(1.85 \mu \mathrm{F} / \mathrm{cm}^{2}\right)$ but less than the cell membrane capacitance of xenopus oocytes (6.3 to $11.9 \mu \mathrm{F} / \mathrm{cm}^{2}$ ) and other excitable cells such as cardiomyocytes $\left(6.83 \mu \mathrm{F} / \mathrm{cm}^{2}\right)$ (Stacey et al., 2014; Polevaya et al., 1999; Methfessel et al., 1986; Kado et al., 1981; Kusano et al., 1982; Gascoyne et al., 2013). In addition, measurements obtained from patch-clamped chromaffin cells showed that the dielectric permittivity of the cell membrane is $\varepsilon_{m e m}=5.7$ (Yoon et al., 2016), a value that agrees to within $20 \%$ with that obtained in this study. Therefore, we conclude that the chromaffin cell membrane capacitance determined in this study using the dielectric spectroscopy technique is reasonably accurate.

Table 2. Measured dielectric properties of chromaffin cells. The properties were calculated using properties listed in table 1 and by fitting experimental data to the granular cell model described in section 2.4.

\begin{tabular}{lcccccc}
\hline & $\begin{array}{c}C_{\text {mem }} \\
\left(\mu \mathrm{F} / \mathrm{cm}^{2}\right)\end{array}$ & $\begin{array}{c}\sigma_{c y t} \\
(\mathrm{~S} / \mathrm{m})\end{array}$ & $\begin{array}{c}C_{g m} \\
\left(\mu \mathrm{F} / \mathrm{cm}^{2}\right)\end{array}$ & $v_{\text {grn }}$ & $\begin{array}{c}\sigma_{g i} \\
(\mathrm{~S} / \mathrm{m})\end{array}$ & $\begin{array}{c}\sigma_{\text {med }} \\
(\mathrm{S} / \mathrm{m})\end{array}$ \\
\hline Mean & 1.22 & 1.14 & 7.10 & 0.44 & 0.35 & 1.35 \\
$\mathrm{SD}$ & 0.16 & 0.25 & 4.04 & 0.08 & 0.12 & 0.07 \\
\hline
\end{tabular}

\subsubsection{Cytoplasmic conductivity}

The cytoplasm dielectric properties $\varepsilon_{c y t}$ and $\sigma_{c y t}$ shown in tables 1 and 2 exclude the effect of the nucleus and large number of secretory granules normally found in the cytosol of chromaffin cells. In order to provide an effective conductivity and permittivity value for the cell interior that takes into account the presence of these structures, the dielectric spectrum of the chromaffin cell interior was calculated using equations (2) to (5) and the results are shown in figure 5.

Figures $5 \mathrm{a}$ and $5 \mathrm{~b}$ show the relative permittivity and conductivity spectra of the chromaffin cell interior, respectively. A dielectric dispersion due to the electrical mismatch between the granular membrane and the cytosol is evident around $5 \mathrm{MHz}$. The relative permittivity of the cell interior (figure 5a) decreases from the low frequency value of 880 to a relative permittivity value that is close to that of water $\left(\varepsilon_{r}=80\right)$ at 20 MHz. This decrease in relative permittivity in the MHz range is due to the charge build-up at the dielectric interfaces in the presence of an alternating electric field, and is explained by the following. A chromaffin 
cell suspension is an electrically heterogeneous system, where the insulating cell membrane separates the conducting cell interior from the extracellular environment. As a result, electrical charges accumulate on the cell membrane and other membranes in the cell interior, forming a large dipole moment at low frequencies. At frequencies much higher than the inverse of the membrane charging time, electric field lines penetrate into the cell interior, thus diminishing these dipoles. Therefore, the low frequency permittivity of the cell interior was measured (i.e., 880) and reflects the presence of the granules and the nucleus. Following dielectric dispersion, this value decreases to 120 at $20 \mathrm{MHz}$.

a

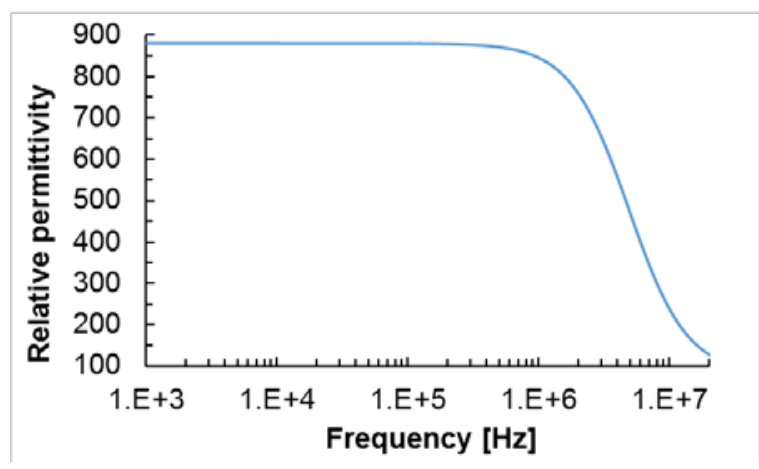

b

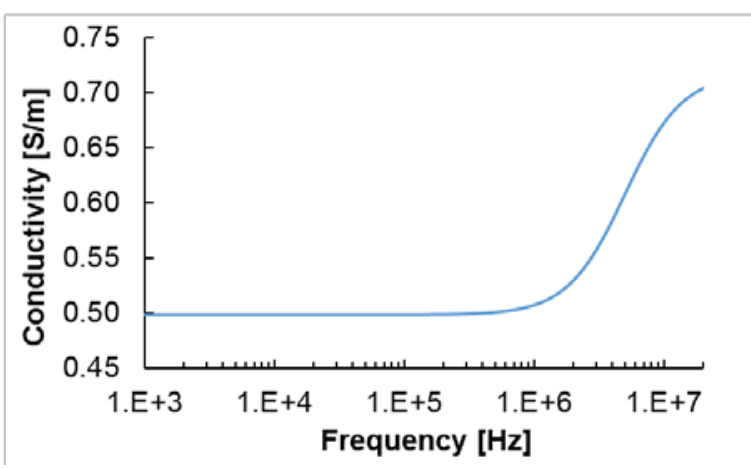

Figure 5 Relative permittivity and conductivity spectra of the chromaffin cell interior. The spectra include the effects of the cytoplasm, nucleus, and granules. The DC conductivity of the cell interior is $0.49 \mathrm{~S} / \mathrm{m}$. Averaged cell properties from three independent experiments in table 2 and values in table 1 were used to calculate the dielectric spectra of the cell interior.

The electrical conductivity of the cell interior reflects the presence of water, ions, granules, and organelles in the cytosol. In this study, a granular cell model was used to model a large volume fraction of granules and the nucleus inside the cell. As shown in figure 5b, the cell interior conductivity increases following the onset (i.e., $400 \mathrm{kHz}$ ) of the dielectric dispersion. The DC conductivity of the cell interior, including the effects of the cytoplasm, nucleus and granules was found to be $0.49 \mathrm{~S} / \mathrm{m}$, which was less than half the cytoplasmic conductivity value of $1.14 \mathrm{~S} / \mathrm{m}$ tabulated in table 2 . This latter value excludes the effects of the membranous structures in the cell interior.

In addition, the cytoplasmic conductivity of various cell types reported in studies that utilized double shell models ranged from $0.12 \mathrm{~S} / \mathrm{m}$ for chondrocytes to $1.31 \mathrm{~S} / \mathrm{m}$ for B lymphocytes (Stacey et al., 2014; 
Polevaya et al., 1999; Zhuang, 2012). These conductivity values include the effects of membranous structures (excluding the nucleus) in the cell interior, which correspond to the conductivity of the intershell space ( $\sigma_{I S}=0.52 \mathrm{~S} / \mathrm{m}$ ) determined in this study, and falls in the range of the conductivity values (0.12 to $1.31 \mathrm{~S} / \mathrm{m})$ reported in the literature. Finally, the hepatocyte cytosol conductivity $\left(\sigma_{c y t}=0.4 \mathrm{~S} / \mathrm{m}\right)$ that was found by Raicu et al. using a vesicular cell model similar to the granular model used in this study was significantly smaller than the cytoplasmic conductivity of $1.14 \mathrm{~S} / \mathrm{m}$ determined for chromaffin cells (Raicu et al., 1998). This difference could be attributed to different ion concentrations in the cytosol of hepatocytes and chromaffin cells. On the other hand, when the effect of the membranous structures in the cytosol of a chromaffin cell (i.e., nucleus and granules) was taken into account, the cytoplasmic conductivity was found to be $0.49 \mathrm{~S} / \mathrm{m}$, lower than the value of $1.14 \mathrm{~S} / \mathrm{m}$ that excludes the effect of the nucleus and granules. These results demonstrate that the ultrastructure of the cell interior plays a role and affects the cytoplasmic conductivity.

\subsubsection{Granule parameters}

The dielectric properties of the granules (i.e., $C_{g m}$ and $\sigma_{g i}$ ) as well as the volume fraction of the granules, $v_{g r n}$, within the cytosol of the cells were calculated using equations (1) to (6) and $C_{g m}=$

$\varepsilon_{g m} \varepsilon_{o} / d_{g r n}$, where $\varepsilon_{g m}$ is the relative permittivity of the granule membrane, $d_{g r n}$ is the granule membrane thickness and $\varepsilon_{o}$ is the permittivity of free space. As previously mentioned, the granular cell model accounts for the presence of numerous granules and a nucleus in the cytoplasm. However, the structure of an actual chromaffin cell is still more complex compared to the model used in this work. Serial electron micrographs of chromaffin cells showed that the ER is localized throughout the cytoplasm, occupying about $14 \%$ of the cell volume (Huh et al., 2005a; Huh et al., 2005b). In addition, in chromaffin cells, the secretory granules and mitochondria are estimated to occupy 20 to 30\% (Plattner et al., 1997; Huh et al., 2005b) and 3\% (Huh et al., 2005b) of the cell volume, respectively. All these membranous structures contribute to the interfacial dispersion observed in the dielectric spectra of chromaffin cell suspensions. In the cell model used in this 
study, all the effects of the cell ultrastructure are lumped into the extracted parameters of the granules. Therefore, it could be possible that the unusually high volume fraction (44\% versus 20 to 30\%) and membrane capacitance of the granules $\left(7.1 \mu \mathrm{F} / \mathrm{cm}^{2}\right)$ reflect the presence of other membranous structures, such as the endoplasmic reticulum and mitochondria in the cell interior. The presence of these intracellular structures in the cytoplasm of a chromaffin cell could affect the extracted cell dielectric parameters in a more complex manner.

\section{Conclusion}

The use of dielectric spectroscopy has gained acceptance as a non-invasive approach for determining the dielectric properties of biological cells. Typically, extraction of cell dielectric properties from measured impedance data is achieved through simplified models for spherical cells, such as the single shell and double shell models. These models are then fit to the dielectric spectrum obtained from impedance measurements of the cell suspension and used to derive the dielectric properties of the cell membrane and nuclear envelope. However, neurosecretory chromaffin cells are characterized by the presence of thousands of membranedelineated secretory granules in their cytosol. Therefore, more sophisticated cell models, as described here, were needed to extract chromaffin cell parameters. Knowledge of these parameters and their incorporation into numerical models will increase our understanding of how the plasma membrane and intracellular organelle membranes of these cells interact with nanosecond electric pulses, which will be important for future applications aimed at modulating neurosecretion.

\section{Acknowledgements}

Research reported in this publication was supported by Grants FA9550-14-1-0018 and FA9550-14-1-0123

from the Air Force Office of Scientific Research, and by the National Institute of Arthritis and Musculoskeletal and Skin Diseases of the National Institute of Health under the award number R21AR063334. The content is solely the responsibility of the authors and does not necessarily represent 
the views of the funding agencies. The authors would like to thank Mike Holcomb at Wolf Pack Meats in Reno, NV for providing fresh bovine adrenal glands. 


\section{References}

Beving H, Eriksson L, Davey C and Kell D 1994 Dielectric properties of human blood and erythrocytes at radio frequencies $(0.2-10 \mathrm{MHz})$; dependence on cell volume fraction and medium composition European biophysics journal 23 207-15

Chang D and Reese T S 1990 Changes in membrane structure induced by electroporation as revealed by rapid-freezing electron microscopy Biophysical journal 58 1-12

Craviso G L 2004 Generation of functionally competent single bovine adrenal chromaffin cells from cell aggregates using the neutral protease dispase Journal of neuroscience methods 137 275-81

Gascoyne P R, Shim S, Noshari J, Becker F F and Stemke-Hale K 2013 Correlations between the dielectric properties and exterior morphology of cells revealed by dielectrophoretic field-flow fractionation Electrophoresis 34 1042-50

Gowrishankar T R, Esser A T, Vasilkoski Z, Smith K C and Weaver J C 2006 Microdosimetry for conventional and supra-electroporation in cells with organelles Biochemical and biophysical research communications $\mathbf{3 4 1} 1266-76$

Hassan N, Chatterjee I, Publicover N G and Craviso G L 2002 Mapping membrane-potential perturbations of chromaffin cells exposed to electric fields IEEE transactions on plasma science 30 1516-24

Huh Y H, Bahk S J, Ghee J Y and Yoo S H 2005a Subcellular distribution of chromogranins A and B in bovine adrenal chromaffin cells FEBS letters 579 5145-51

Huh Y H, Yoo J, Bahk S J and Yoo S H 2005b Distribution profile of inositol 1, 4, 5-trisphosphate receptor isoforms in adrenal chromaffin cells FEBS letters 579 2597-603

Irimajiri A, Suzaki T and Asami K 1991 Dielectric Modeling of Biological Cells: Models and Algorithm Bull. Inst. Chem. Res., Kyoto Univ 69 421-438

Kado R T, Marcher K and Ozon R 1981 Electrical membrane properties of the Xenopus laevis oocyte during progesterone-induced meiotic maturation Developmental biology 84 471-6

Koklu A, Sabuncu A C and Beskok A 2016 Rough Gold Electrodes for Decreasing Impedance at the Electrolyte/Electrode Interface Electrochimica Acta 205 215-25

Kusano K, Miledi R and Stinnakre J 1982 Cholinergic and catecholaminergic receptors in the Xenopus oocyte membrane The Journal of physiology 328143

Methfessel C, Witzemann V, Takahashi T, Mishina M, Numa S and Sakmann B 1986 Patch clamp measurements onXenopus laevis oocytes: currents through endogenous channels and implanted acetylcholine receptor and sodium channels Pflügers Archiv European Journal of Physiology 407 577-88

Pajkossy T 1994 Impedance of rough capacitive electrodes Journal of Electroanalytical Chemistry 364 111-125

Pakhomov A G, Kolb J F, White J A, Joshi R P, Xiao S and Schoenbach K H 2007a Long-lasting plasma membrane permeabilization in mammalian cells by nanosecond pulsed electric field (nsPEF) Bioelectromagnetics 28 655-663

Pakhomov A G, Shevin R, White J A, Kolb J F, Pakhomova O N, Joshi R P and Schoenbach K H 2007b Membrane permeabilization and cell damage by ultrashort electric field shocks Archives Biochemistry Biophysics 465 109-118

Pakhomov A G, Bowman A M, Ibey B L, Andre F M, Pakhomova O N and Schoenbach K H 2009 Lipid nanopores can form a stable, ion channel-like conduction pathway in cell membrane Biochemical and Biophysical Research Communications 385 181-186

Plattner H, Artalejo A R and Neher E 1997 Ultrastructural organization of bovine chromaffin cell cortex-analysis by cryofixation and morphometry of aspects pertinent to exocytosis The Journal of cell biology 139 1709-17

Polevaya Y, Ermolina I, Schlesinger M, Ginzburg B-Z and Feldman Y 1999 Time domain dielectric spectroscopy study of human cells: II. Normal and malignant white blood cells Biochimica et Biophysica Acta (BBA)-Biomembranes 1419 257-71 
Raicu V, Saibara T, Enzan H and Irimajiri A 1998 Dielectric properties of rat liver in vivo: analysis by modeling hepatocytes in the tissue architecture Bioelectrochemistry and bioenergetics 47 333-42

Sabuncu A C, Zhuang J, Kolb J F and Beskok A 2012 Microfluidic impedance spectroscopy as a tool for quantitative biology and biotechnology Biomicrofluidics 6034103

Scarlett S S, White J A, Blackmore P F, Schoenbach K H and Kolb J F 2009 Regulation of intracellular calcium concentration by nanosecond pulsed electric fields Biochimica et Biophysica Acta (BBA) - Biomembranes 1788 1168-75

Schoenbach K H, Beebe S J and Buescher E S 2001 Intracellular effect of ultrashort electrical pulses Bioelectromagnetics 22 440-8

Schwan H 1968 Electrode polarization impedance and measurements in biological materials Annals of the New York Academy of Sciences 148 191-209

Semenov I, Xiao A and Pakhomov A G 2013a Primary pathways of intracellular $\mathrm{Ca}^{2+}$ mobilization by nanosecond pulsed electric field Biochimica Biophysica Acta (BBA) - Biomembranes 1828 981989

Semenov I, Xiao S, Pakhomova O N and Pakhomov A G 2013b Recruitment of the intracellular $\mathrm{Ca}^{2+}$ by ultrashort electric stimuli: The impact of pulse duration Cell Calcium 54 145-150

Smith K C and Weaver J C. 2008 Active mechanisms are needed to describe cell responses to submicrosecond, megavolt-per-meter pulses: Cell models for ultrashort pulses Biophysical Journal 95 1547-1563

Stacey M W, Sabuncu A C and Beskok A 2014 Dielectric characterization of costal cartilage chondrocytes Biochimica et Biophysica Acta (BBA)-General Subjects 1840 146-52

Vernier P T, Sun Y and Gundersen M A 2006 Nanoelectropulse-driven membrane perturbation and small molecule permeabilization BMC cell biology 737

Waymire J C, Bennett W F, Boehme R, Hankins L, Gilmer-Waymire K and Haycock J W 1983 Bovine adrenal chromaffin cells: high-yield purification and viability in suspension culture Journal of neuroscience methods 7 329-51

Yoon J, Leblanc N, Zaklit J, Vernier P T, Chatterjee I and Craviso G L 2016 Enhanced Monitoring of Nanosecond Electric Pulse-Evoked Membrane Conductance Changes in Whole-Cell Patch Clamp Experiments The Journal of membrane biology 249 633-44

Zaklit J, Yoon J, Chatterjee I, Evans E, Vernier P T, Leblanc N and Craviso G L $2015 \mathrm{Ca}^{2+}$ release from internal stores of adrenal chromaffin cells is not evoked by 5 ns electric pulses Proceedings of the Annual Meeting of the Bioelectromagnetics Society, (Asilomar, CA)

Zaklit J, Craviso G L, Leblanc N, Yang L, Vernier P T and Chatterjee I 2017 Adrenal chromaffin cells exposed to 5-ns pulses require higher electric fields to porate intracellular membranes than the plasma membrane: an experimental and modeling study Under review

Zhuang J 2012 Nonosecond pulsed electric field induced changes in dielectric properties of biological cells. (PhD Dissertation, Norfolk, VA: Old Dominion University) 
$\mathbf{a}$

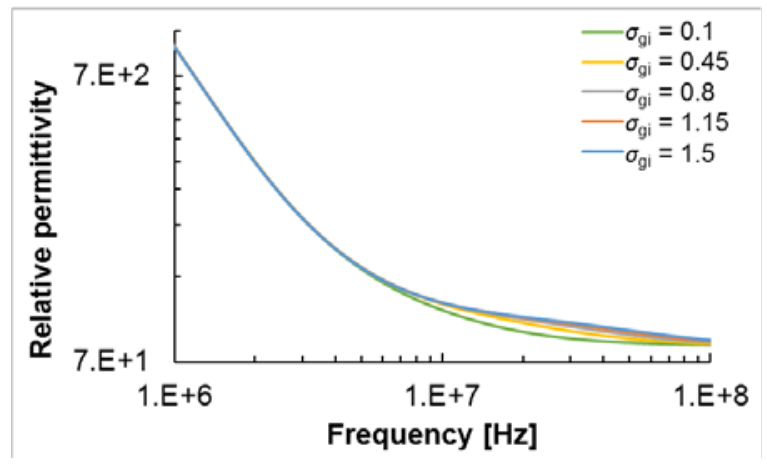

b

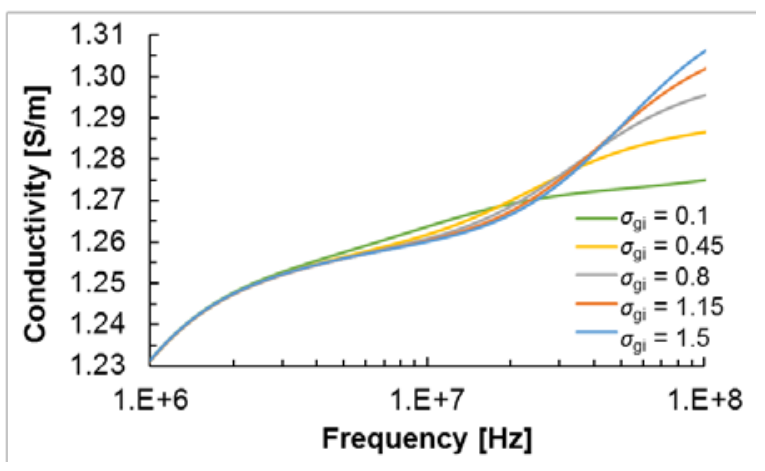

Figure S1 The small changes in figures $4 \mathrm{a}$ and $4 \mathrm{~b}$ showing the frequency dependent variations in the granule milieu relative permittivity and conductivity are detailed in (a) and (b), respectively. 